\title{
Wearable multimode sensor with a seamless integrated structure for recognition of different joint motion states with the assistance of a deep learning algorithm
}

\author{
Lei Wen ${ }^{1}$, Meng Nie $\mathbb{0}^{1 凶}$, Pengfan Chen ${ }^{1}$, Yu-na Zhao ${ }^{1}$, Jingcheng Shen ${ }^{1}$, Chongqing Wang ${ }^{1}$, Yuwei Xiong ${ }^{1}$,
} Kuibo Yin ${ }^{1}$ and Litao Sun (1) ${ }^{1 凶}$

\begin{abstract}
Accurate motion feature extraction and recognition provide critical information for many scientific problems. Herein, a new paradigm for a wearable seamless multimode sensor with the ability to decouple pressure and strain stimuli and recognize the different joint motion states is reported. This wearable sensor is integrated into a unique seamless structure consisting of two main parts (a resistive component and a capacitive component) to decouple the different stimuli by an independent resistance-capacitance sensing mechanism. The sensor exhibits both high strain sensitivity ( $G F=7.62,0-140 \%$ strain) under the resistance mechanism and high linear pressure sensitivity $\left(S=3.4 \mathrm{kPa}^{-1}, 0-14 \mathrm{kPa}\right.$ ) under the capacitive mechanism. The sensor can differentiate the motion characteristics of the positions and states of different joints with precise recognition (97.13\%) with the assistance of machine learning algorithms. The unique integrated seamless structure is achieved by developing a layer-bylayer casting process that is suitable for large-scale manufacturing. The proposed wearable seamless multimode sensor and the convenient process are expected to contribute significantly to developing essential components in various emerging research fields, including soft robotics, electronic skin, health care, and innovative sports systems applications.
\end{abstract}

\section{Introduction}

Monitoring and recognizing motion features of the human body with wearable sensors are essential functions for understanding human activities and vital signs, especially in applications such as intelligent medical rehabilitation, smart sport exercise, soft robotics, and electronic $\operatorname{skin}^{1-6}$. However, human motion is a complex process involving multiple joints, and each joint motion consists of both the bending/compressing of the skeleton and the stretching of the skin covered in the surface of the bone. To date, typical flexible sensors usually contact the

\footnotetext{
Correspondence: Meng Nie (m_nie@seu.edu.cn) or Litao Sun (slt@seu.edu.cn) 'SEU-FEl Nano-Pico Center, Key Laboratory of MEMS of Ministry of Education, School of Electronic Science \& Engineering, Southeast University, Nanjing 210096, P. R. China
}

surface of human bodies; thus, these sensors are stimulated by both compressive pressure and stretching strain. Under different stimuli, the majority of sensors yield similar electrical output, making it challenging to distinguish these mechanical inputs ${ }^{7-9}$. Because the bending/ compression and stretching of each joint occur together, it is highly necessary to develop a multimode wearable sensor to monitor and decouple the multiple parameters of the joint's motions ${ }^{10,11}$.

In recent years, three strategies have been proposed to design multimode flexible sensors. The first is to design a single structure sensor to monitor different stimuli simultaneously ${ }^{12-15}$. Song et al. deposited multiwall carbon nanotubes (MWCNTs) onto a wrinkled fabric surface to measure tangential stretching forces and 
normal pressure with opposite resistance responses ${ }^{16}$. Qin et al. proposed a multifunctional epidermal sensor by immersing hydrophobic carbon nanotubes (CNTs) into a polyacrylamide hydrogel, which exhibited high tensile strain sensitivity in wide strain ranges and high linear sensitivity in a large pressure region ${ }^{17}$. Chao et al. presented a multifunctional skin-like sensor through a dopamine-triggered gelation route. The sensor was sensitive to strain, pressure, and temperature ${ }^{18}$. However, it remains difficult for a single-structure strategy to distinguish different stimuli. The second strategy is to design two discrete sensors and assemble them together in a certain way to detect different stimuli ${ }^{19,20}$. Su et al. applied $\mathrm{NaCl}$-doped agarose gel as a biocompatible conductive filler and injected it into a 3D printed elastomer shaper for wearable sensors. The sensors exhibited the ability to differentiate bending and stretching motions by assembling both straight and spring channels inside the elastomer shaper ${ }^{21}$. Lee et al. fabricated multidirectional strain sensors by cross-compacting two anisotropic carbon nanofiber sensors together, which realized stretching strains parallel and perpendicular to sensor alignment ${ }^{22}$. These assembled sensors solved the decoupling problem of multiple stimuli well. However, the mechanical properties and stability are always challenging, and the manufacturing process is relatively complicated. The third is to design a single structure as the sensor with two integrated independent parts ${ }^{23}$. Park et al. presented stretchable energy harvesting e-skin, which consisted of PDMS/SWNT-film/porous PDMS/ PDMS spacers/SWNT-film/PDMS (from top to bottom). The sensor can detect and differentiate pressure and strain signals simultaneously ${ }^{24}$. However, the complicated process flow increased both the difficulty of fabrication and the cost of the sensor. It is critical to develop a wearable multimode sensor with convenient manufacturing to decouple multiple parameters.

In this study, we present a wearable seamless resistancecapacitance structural multimode (SRCSM) sensor that can decouple the pressure and stretchable strain applied on each joint during human motion. The SRCSM sensor is integrated into a unique seamless structure, which consists of two different main parts (a resistive component and a capacitive component) to decouple the different stimuli by an independent resistance-capacitance sensing mechanism. A layer-by-layer casting process is proposed, which is convenient and suitable for large volume manufacturing. Furthermore, the SRCSM sensor can differentiate the motion characteristics of the positions and states of different joints with precise recognition (97.13\%) with the assistance of machine learning algorithms. These demonstrations indicate that the proposed SRCSM sensor and processing technique have high potential value in various emerging research fields, including soft robotics, health care monitoring, and intelligent sports systems applications.

\section{Results and discussion \\ Fabrication of the SRCSM sensor}

The layer-by-layer casting processable fabrication of the SRCSM sensor is schematically illustrated in Fig. 1. First, a polyvinyl chloride (PVC) mold $(600 \mu \mathrm{m}$ in depth, fabricated by laser cutting) was attached to a polyethylene terephthalate (PET) substrate. Parts A and B of

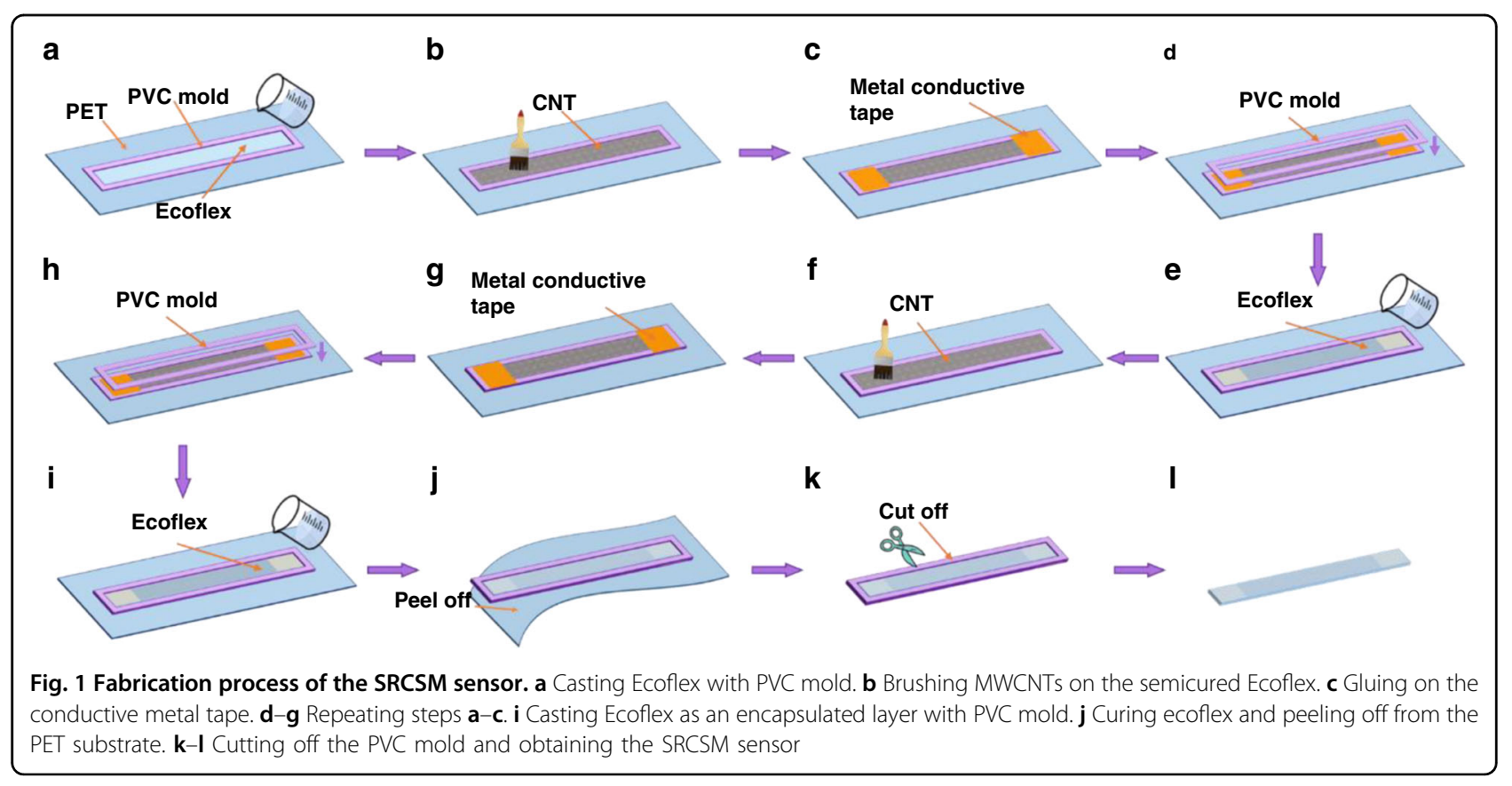


Ecoflex were mixed at a weight ratio of $1: 1$ and then poured into the PVC mold after the bubbles were dissipated. The solutions were cured for $50 \mathrm{~min}$ to obtain the semicured Ecoflex (Fig. 1a). Second, the MWCNTs $\left(1 \mathrm{mg} / \mathrm{cm}^{2}\right)$ were pressed into the surface of semicured Ecoflex by brushing. Due to the pressure during brushing, part of the MWCNTs were embedded into the incompletely cured Ecoflex. (Fig. 1b). After 3 hours of full curing, a hybrid MWCNT/Ecoflex thin film layer formed on the surface of Ecoflex with some MWCNTs embedded into the surface of Ecoflex. Conductive metal tapes were attached to the hybrid MWCNT/Ecoflex thin film layer as the lead electrodes (Fig. 1c). Then, the above steps were repeated twice, as shown in Fig. 1d to g. Subsequently, the casting process of Ecoflex was repeated to encapsulate the structure (Fig. 1i). Ecoflex was fully cured for 3 hours and peeled off from the PET substrate, as illustrated in Fig. 1j. Finally, the PVC mold was cut off, and the SRCSM sensor was obtained, as shown in Fig. 1k, l. All fabrication steps were completed at room temperature $\left(20^{\circ} \mathrm{C}, 50 \%\right.$ humidity $)$.

\section{Morphology characterization of the SRCSM sensor}

Figure 2 shows the conceptual structural design and morphology characterization for the proposed SRCSM sensor. As shown in Fig. 2a, the two facesheets of the sandwich-shaped sensor made of hybrid Ecoflex/ MWCNT films served as the electrodes, and pure Ecoflex served as the dielectric layer for the capacitive component. Copper tapes were attached to the facesheets for measuring output signals. Two Ecoflex layers were used to encapsulate the sensor. Additionally, the facesheet of the electrode was utilized as an independent resistive component. Figure $2 \mathrm{~b}$ shows the appearance of the SRCSM sensor. Figure $2 \mathrm{c}-\mathrm{e}$ shows the cross-sectional photography and SEM images of the proposed sensor. The thickness of each electrode plate is approximately $25 \mu \mathrm{m}$, the dielectric layer is approximately $1.2 \mathrm{~mm}$, and the encapsulation layer is $600 \mu \mathrm{m}$. Figure $2 \mathrm{f}-\mathrm{h}$ displays the microsurface topography of the electrodes, which is similar to our previous research ${ }^{25}$. A brush was used to press MWCNTs into the semicured Ecoflex surface. Under the action of force, the MWCNTs were embedded in the Ecoflex surface and formed a hybrid conductive layer, which was used as the electrode. Figure $2 \mathrm{f}-\mathrm{h}$ also shows that MWCNTs are evenly distributed on Ecoflex. Furthermore, due to the discrete nature of the bristles, a microstructure with peaks and valleys was formed on the surface of the hybrid layer during the brushing process. Thus, the resistive component can be regarded as a double-layer structure: one is the peak-valley microstructured hybrid layer, and the other is the flat hybrid layer at the bottom of the valley. The length and width directions of the sensor are denoted as the $\mathrm{x}$-axis and $y$-axis, and the through-thickness direction is represented as the $\mathrm{z}$-axis. The resistance $(R)$ and capacitance $(C)$ values of the SRCSM sensor can be simultaneously changed under mechanical stimuli such as in-plane stretching strain $(\varepsilon)$ in the $\mathrm{x}$-axis or through-thickness compressive pressure $(p)$ in the $\mathrm{z}$-axis.

\section{Mechanical, electrical property and sensing mechanism of the SRCSM sensor}

To quantitatively evaluate the mechanical properties, stretching and compressive tests were investigated. Figure 3a illustrates the stretching stress-strain curves of the SRCSM sensor and the pure Ecoflex slender (the stretching rate was $10.1 \mathrm{~mm} / \mathrm{min}$ ). The Young's modulus of the seamless structure sensor is $269 \mathrm{kPa}$, and the fracture strain is $458 \%$. The identical tests were given to the neat Ecoflex slender with the same geometries to make comparisons. The Young's modulus of the pure Ecoflex slender is $77 \mathrm{kPa}$, and the fracture strain is $469 \%$. Introducing hybrid Ecoflex/MWCNT electrode layers has a specific toughening effect on the seamless structure and has little effect on the fracture strain. Similarly, the mechanical compressive properties of the SRCSM sensor were also evaluated by compressive models (the compressive rate was $0.5 \mathrm{~mm} / \mathrm{min}$ ). The compressive stress-strain curves of the SRCSM sensor are shown in Fig. 3b. Representative stretching loading-unloading tests at $120 \%$ strain are displayed in Fig. 3c. All hysteresis loops remained almost unchanged during 15 cycles, indicating the excellent fatigue resistance of the SRCSM sensor due to the elastic characteristics. In addition, cyclic compressive tests at $17.5 \%$ strain (ca. $14 \mathrm{kPa}$, compressive strength) revealed the excellent elasticity of the SRCSM sensor (Fig. 3d). Such stable and tough mechanical characteristics of the SRCSM sensor are realized due to the overall seamless structure rather than two independent sensing devices assembled.

The direct current-voltage characteristic curves of the SRCSM sensor under different applied stretching strains and pressures are also shown in Fig. 3e and $f$. The currents were recorded in the voltage range of -1 to $1 \mathrm{~V}$. The high linear behaviors of the curves demonstrate that the device has an excellent ohmic contact, and the reciprocal slope of the $I-V$ curves can be used to estimate the resistance of the sensor. As shown in the insets in Fig. 3e, the average resistances under different stretching strains of $0 \%, 30 \%$, $60 \%, 90 \%$, and $120 \%$ are $22 \mathrm{k} \Omega, 30.4 \mathrm{k} \Omega, 46.5 \mathrm{k} \Omega, 75.3 \mathrm{k} \Omega$, and $120.6 \mathrm{k} \Omega$, respectively. This indicates that the resistance of the electrode increases rapidly with the applied strain. Similarly, the inset in Fig. 3f illustrates that the resistances are $22 \mathrm{k} \Omega, 22.1 \mathrm{k} \Omega, 22.4 \mathrm{k} \Omega$ and $23.7 \mathrm{k} \Omega$ under $0 \mathrm{kPa}, 2 \mathrm{kPa}, 4 \mathrm{kPa}$, and $14 \mathrm{kPa}$, respectively. Compared with stretching stimuli, the resistance of the sensor under different pressure stimuli is relatively stable. 


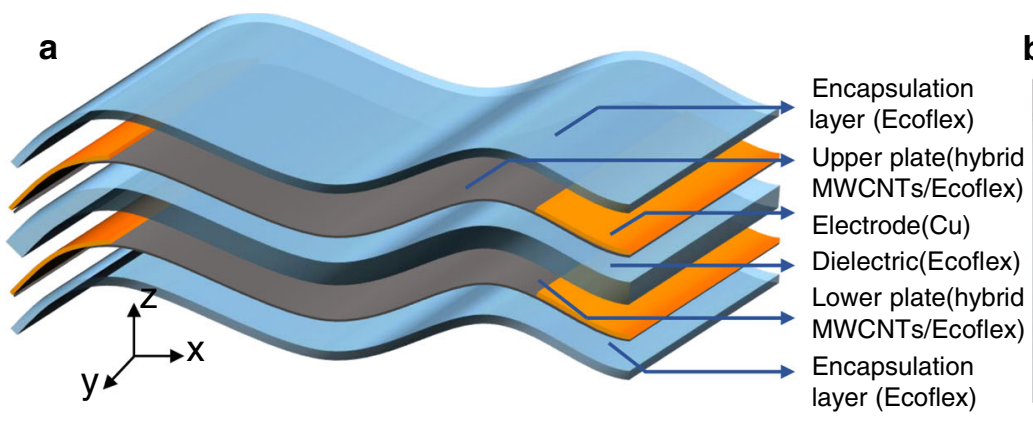

b
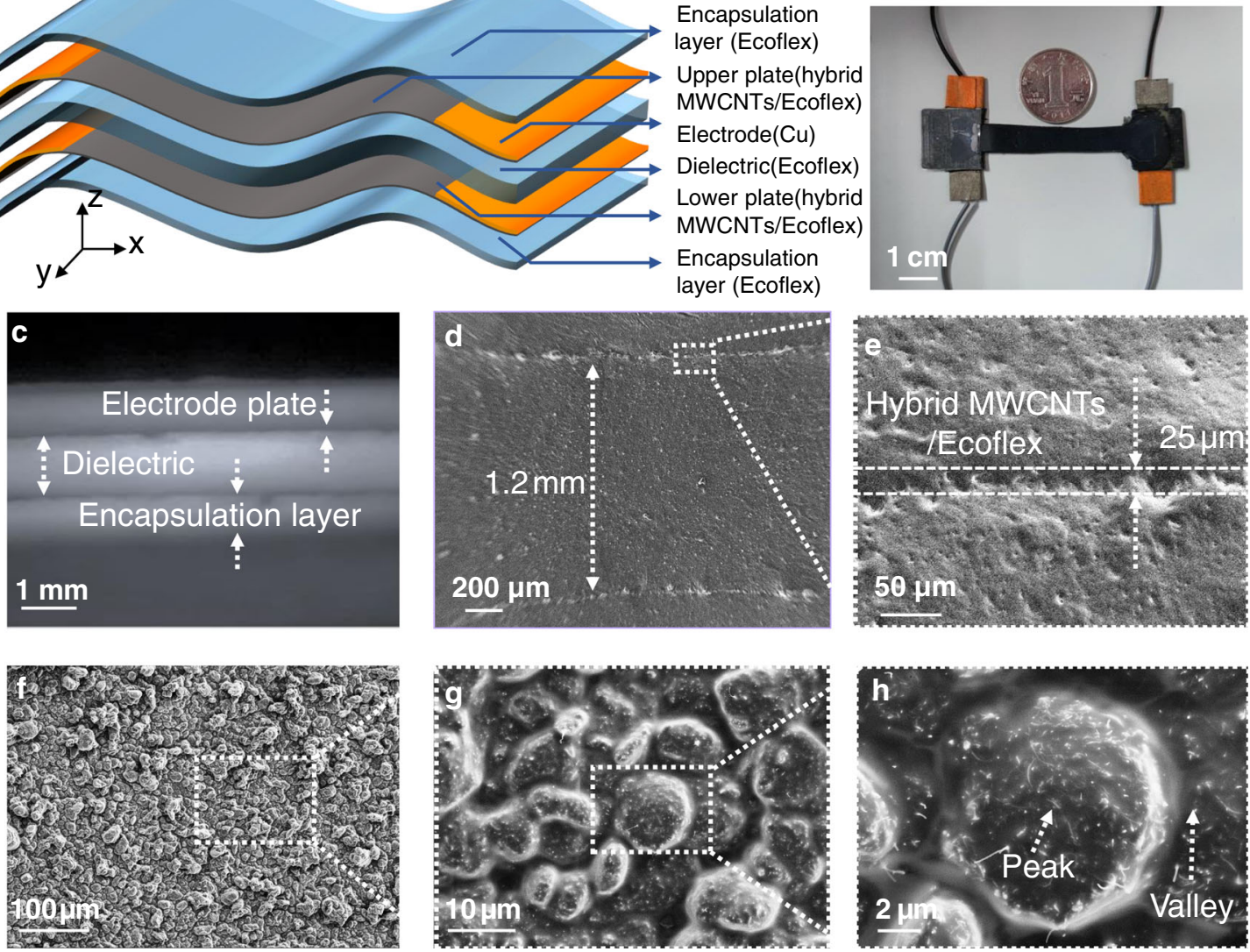

Fig. 2 Morphology characterization of the SRCSM sensor a Exploded view of the SRCSM sensor. $\mathbf{b}$ Photograph of the integrated SRCSM sensor. c-e Cross-sectional photograph and SEM images of the SRCSM sensor. $\mathbf{f}-\mathbf{h}$ SEM surface topography of the SRCSM sensor

The bias-free resistance will simplify the relationship between the output electrical signals and the applied strains and the data process integrated circuit.

The gauge factor of the sensor is an important parameter to evaluate the sensing performance of the device. It is usually defined as $\mathrm{GF}=\left(\Delta R / R_{0}\right) / \varepsilon$, where $R_{0}$ is the initial resistance, $\Delta R$ denotes the change in the resistance before and after the application of strain, and $\varepsilon$ represents the applied strain. Figure $4 \mathrm{a}$ indicates the typical relationship between the relative resistance variation ratio $\left(\Delta R / R_{0}\right)$ and the applied stretching strain $\varepsilon$ (up to $140 \%$ ) of the sensor at a stretching rate of $10.1 \mathrm{~mm} / \mathrm{min}$. The characteristic curve can be divided into two distinct regions with GF values of $2.02(0<\varepsilon<80 \%)$ and 7.62 (80-140\%), which depend on the peak-valley synergetic conductive paths in the resistive structure. To further reveal the sensing mechanism from electrical modeling, the resistance $R$ was decomposed into three parts: $R_{1}, R_{2}$, and $R_{3}$, representing the resistance of the slope of the peak, the bottom of the valley and the interior of the peak, respectively. The detailed electrical model is shown in Fig. 4c. To simplify the circuit and facilitate the calculation, the circuit is equivalent based on Thevenin's theorem, as shown in Fig. 4d. The overall equivalent circuit resistance is described as:

$$
\begin{aligned}
& R=R_{12}+\frac{\left(R_{13}+R_{1}\right) *\left(R_{23}+R_{2}\right)}{R_{13}+R_{1}+R_{23}+R_{2}} \\
& R_{12}=\frac{R_{1} * R_{2}}{R_{1}+R_{2}+R_{3}}, R_{13}=\frac{R_{1} * R_{3}}{R_{1}+R_{2}+R_{3}}, R_{23}=\frac{R_{2} * R_{3}}{R_{1}+R_{2}+R_{3}}
\end{aligned}
$$

In region I $(0<\varepsilon<80 \%)$, the bottom of the valley was stretched first, causing a large number of conductive paths to be destroyed, and $R_{2}$ increased sharply and almost infinitely. However, the slope of the peak becomes slow under stretching strain, and the interior of the peak is squeezed, resulting in a slight decrease in $R_{3}$ and an almost unchanged $R_{1}$. The resistance $R$ can be approximated as the series resistance of $R_{12}, R_{13}$ and $R_{1}$, where $R_{12}$ increases with the increase of $R_{2}$, and $R_{13}$ decreases with the increase of $R_{2}$, making the change of resistance in this region relatively small. As the applied strain gradually 

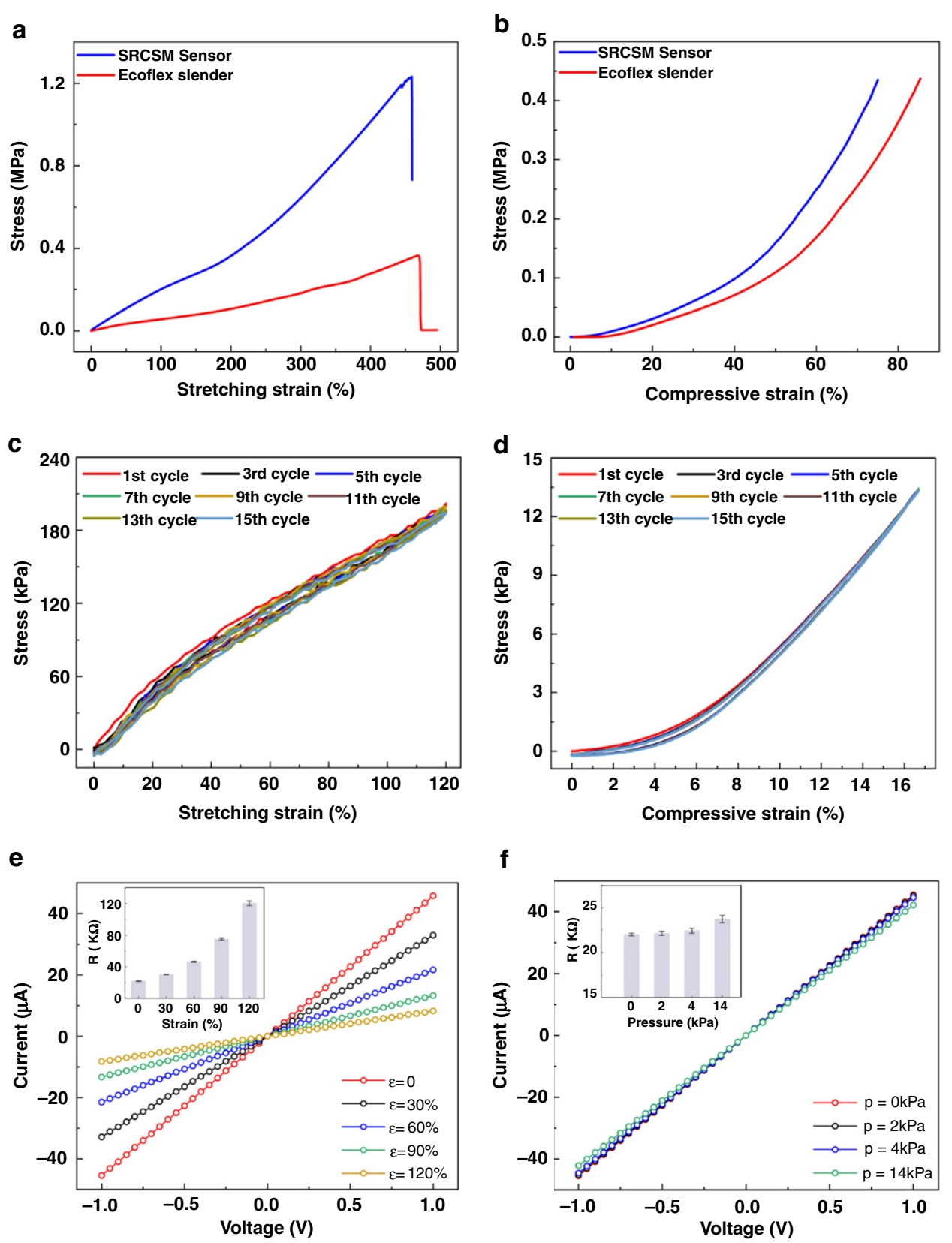

Fig. 3 Mechanical and electrical properties of the SRCSM sensor. a Stretching stress-strain curves of the SRCSM sensor and the pure slender Ecoflex. $\mathbf{b}$ The compressive stress-strain curves of the SRCSM sensor and pure slender Ecoflex. $\mathbf{c}$ Fifteen successive cyclic loading-unloading curves of the SRCSM sensor at a maximum stretching strain of 120\%. d Fifteen successive cyclic loading-unloading curves of the SRCSM sensor at $17.5 \%$ strain (ca. $14 \mathrm{kPa}$, compressive strength). e The curves under various stretching stains. $\mathbf{f}$ The curves under various pressures. The applied voltage is from -1 $\mathrm{V}$ to $1 \mathrm{~V}$. (Insets: The average resistance $(R)$ under different applied stimuli)

increased to region II (80-140\%), the peak was gradually stretched, and the peak-valley microstructures almost disappeared, as shown in the insets of Fig. 4a. Therefore, the resistance $R_{1}$ increased drastically with increasing strain, and $R_{2}$ and $R_{3}$ remained almost unchanged. The resistance of $R_{12}$ and $R_{13}$ both increased with the increase of $R_{1}$, making a higher sensitivity in this region.
The sensitivity $S$ of the sensor under pressure is defined as $S=\left(\left(\Delta R / R_{0}\right) / \Delta p\right)$, where $\Delta p$ represents the value of applied pressure. Figure $4 \mathrm{~b}$ indicates the typical relationship between the relative resistance variation ratio $\left(\Delta R / R_{0}\right)$ and the applied pressure (up to $14 \mathrm{kPa}$ ) of the sensor at a pressing rate of $0.5 \mathrm{~mm} / \mathrm{min}$. The resistance variation ratio $\left(\Delta R / R_{0}\right)$ increases with the applied 


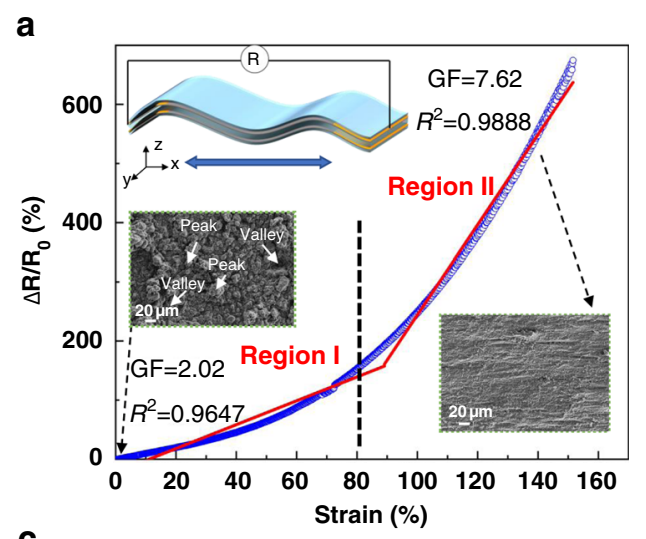

C

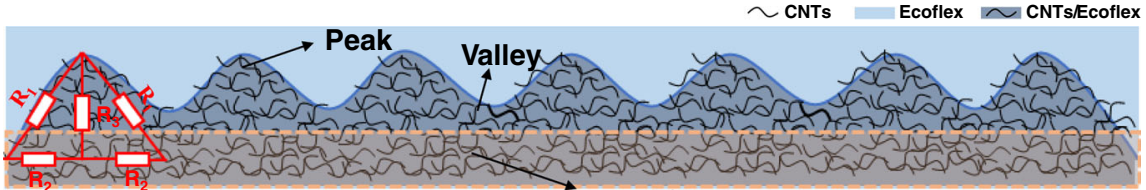

d

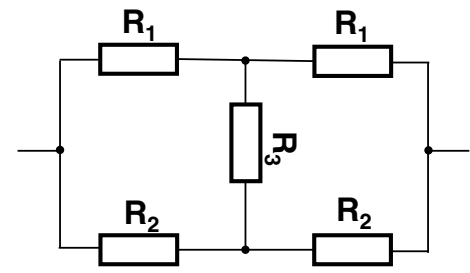

$\mathbf{e}$

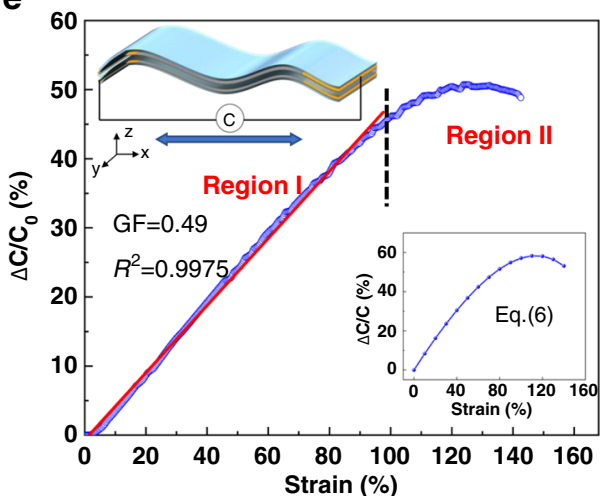

b

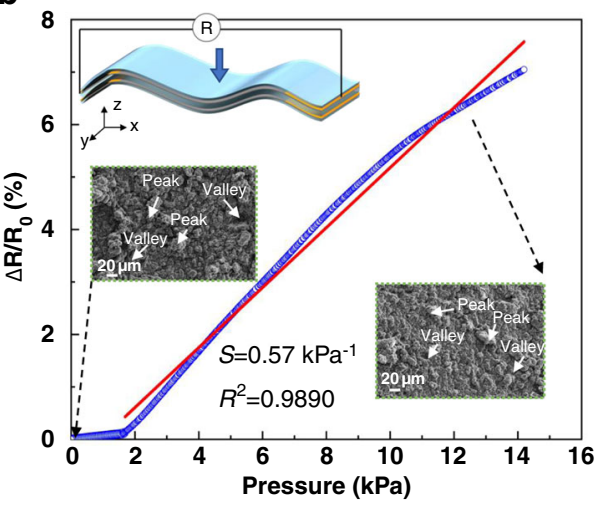

Bottom of the valley

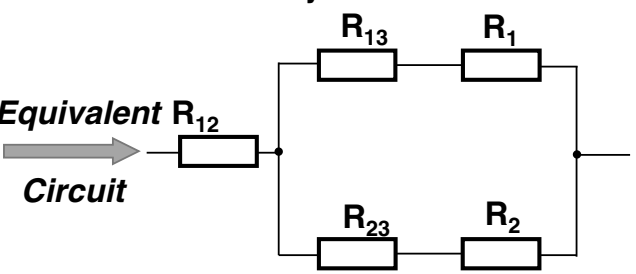

f

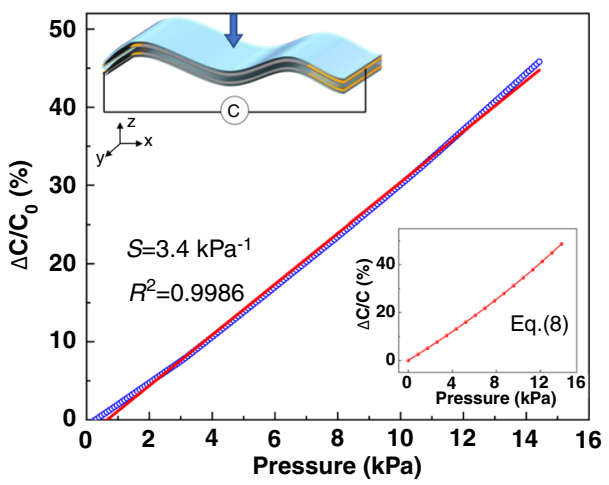

Fig. 4 Sensing performance of the SRCSM sensor. $\mathbf{a}$, $\mathbf{b}$ Relative resistance variation ratio $\left(\triangle R / R_{0}\right)$ as a function of the applied stretching strain and pressure. The insets are SEM surface topographies of the SRCSM sensor under the different stimuli. c The electrical modeling of the SRCSM sensor. d The transformation of the $\Delta-Y$ equivalent circuit based on Thevenin's theorem. $\mathbf{e}$, $\mathbf{f}$ Relative capacitance variation ratio $\left(\Delta C / C_{0}\right)$ as a function of the applied stretching strain and pressure. The blue dots are experiments results. The solid red lines are the corresponding linear fitting lines, and the fitting coefficients are also indicated. The insets: Theoretical curves

pressure, leading to tangential stretching of the resistive structure. The SRCSM sensor obtains a small $S$ of nearly $0.57 \mathrm{kPa}^{-1}$ due to slight tangential stretching. The slow increase in the range of $0-2 \mathrm{kPa}$ is due to the slight decrease in $R_{3}$ under pressure, which suppresses the increase in resistance.

Figure $4 \mathrm{e}$ indicates the relationship between the capacitance variation ratio $\Delta C / C_{0}$ and the applied stretching strain $\varepsilon$ (up to $140 \%$ ) of the sensor at a stretching rate of $10.1 \mathrm{~mm} / \mathrm{min}$. For the capacitance strain sensor, GF can be defined as GF $=\left(\Delta C / C_{0}\right) / \varepsilon$, where $C_{0}$ is the initial capacitance and $\Delta C$ denotes the capacitance change before and after the application of strain. The curve can also be divided into two regions. In region $\mathrm{I}(0<\varepsilon<100 \%)$, the sensor obtained a linear characteristic curve, and after that, the capacitance variation ratio $\Delta C / C_{0}$ decreased. The electrical model of the sensor is constructed to reveal the working mechanism. The parallel plate capacitance $C$ can be defined as $C \equiv Q / V$, where $Q$ is the stored charge and $V$ is the electrostatic potential. Ignoring the influence of 
fringe field effects and according to Gauss's theorem, the magnitude of electric field $E$ is related to charge $Q$, which can be obtained as $E=Q / \varepsilon_{\mathrm{r}} A$. $\varepsilon_{\mathrm{r}}$ is the relative permittivity, and $A$ is the area of the capacitor. Since the voltage value is equal to the product of electric field $E$ and electrode plate spacing $d$, the parallel plate capacitance $C$ is:

$$
C \equiv \frac{Q}{V}=\frac{Q}{E d}=\frac{Q}{\frac{Q}{\varepsilon_{r} A} d}=\frac{\varepsilon_{r} A}{d}
$$

Equation (3) shows that the capacitance $C$ has no relationship with the amount of charge $Q$ stored in the electrode plate, so the resistance change of the electrode plate can be ignored.

To verify the accuracy of the experimental results under the applied strain, we deduced the formula of the variation ratio of capacitance. The change in the capacitance $\Delta C$ can be defined as $\Delta C=C-C_{0}$, where $C_{0}$ is the initial capacitance and $C$ is the capacitance under the applied strain. According to Eq. (1), the capacitance $C$ can be expressed as:

$$
C=\frac{\varepsilon_{r}\left(w_{0}+\triangle w\right)\left(l_{0}+\triangle l\right)}{d_{0}+\triangle d}
$$

where $\Delta l, \Delta w$, and $\Delta d$ represent the variation of the length, width, and thickness of the device under the applied strain, respectively. According to the calculated formula of Poisson's ratio (detailed formula derivation can be achieved in the support information in Part 1), the variation ratio of capacitance $\Delta C / C_{0}$ can also be defined as:

$$
\frac{\triangle C}{C_{0}}=\frac{\left(1-\frac{\Delta l}{l_{0}} * \nu_{x y}\right)\left(1+\frac{\Delta l}{l_{0}}\right)}{1-\frac{\triangle l}{l_{0}} * \nu_{x z}}-1
$$

$\Delta l / l_{0}$ can be written as $\varepsilon$, which represents the stretching strain:

$$
\frac{\triangle C}{C_{0}}=\frac{\left(1-\varepsilon * \nu_{x y}\right)(1+\varepsilon)}{1-\varepsilon * \nu_{x z}}-1
$$

where $v_{x y}$ and $v_{x z}$ are Poisson's ratios in the $y$ and $z$ directions, respectively. Poisson's ratios $v_{\mathrm{xy}}$ and $v_{\mathrm{xz}}$ of the structure are 0.45 and 0.3 , respectively, which can be calculated by measured data, as shown in Fig. S1. The inset in Fig. 4e shows the functional relationship between the variables $\Delta l / \mathrm{l}_{0}$ and $\Delta C / C_{0}$. In region I $(0<\varepsilon<100 \%)$, as the stretching strain increases, the length of the electrode plate increases, the area of the electrode plate increases, and the distance between the electrode plates decreases. Therefore, the capacitance increases with the strain, and the GF value is 0.49 . In region II $(100 \%<\varepsilon<$ $140 \%)$, as the strain increases to a certain extent, the decrease in width cannot be ignored anymore, resulting in a relatively small increase or even decrease in the area of the electrode plate. The theoretical derivation result shows the same characteristics as the experimental results in Fig. 4e.

The typical relationship between the capacitance variation ratio $\Delta . C / C_{0}$ and the applied pressure of the sensor is shown in Fig. 4f. The sensitivity of the capacitance pressure sensor can be defined as $S=\left(\left(\Delta C / C_{0}\right) / \Delta p\right)$, where $\Delta p$ represents the value of applied pressure and $C_{0}$ represents the original capacitance of the sensor. The $S$ value of the capacitance pressure sensor is $3.4 \mathrm{kPa}^{-1}$. As the pressure increases, $d$ of the capacitance decreases, $A$ of the plates increases, and the capacitance $C$ increases sequentially. To verify the linearity of the capacitance variation ratio pressure curves, the equation of $\Delta . C / C_{0}$ under pressure is also derived (the detailed formula derivation can be achieved in support information Part. 2). The variation ratio of capacitance $\Delta C / C_{0}$ is defined as:

$$
\begin{aligned}
& \frac{\triangle C}{C_{0}}=\frac{\left(1+\frac{\Delta d}{d_{0}} * \nu_{z x}\right)\left(1+\frac{\triangle d}{d_{0}} * \nu_{z y}\right)}{1-\frac{\triangle d}{d_{0}}}-1 \\
& \frac{\triangle C}{C_{0}}=\frac{\left(1+\lambda * \triangle p * \nu_{z x}\right)\left(1+\lambda * \triangle p * \nu_{z y}\right)}{1-\lambda * \triangle p}-1
\end{aligned}
$$

where $\lambda$ represents the relation between the strain $\Delta d / d_{0}$ and the applied pressure $\Delta p$, and the expression can be obtained by fitting the compressive stress-strain curve of Fig. 3d. Poisson's ratios $v_{z x}$ and $v_{z y}$ of the structure are both nearly 0.66 , which can be calculated from Fig. S1. The theoretical derivation result of the capacitance variation ratio-pressure curves is shown in the inset in Fig. 4f, which is consistent with the experimental result.

The sensitivity $S$ was converted to the GF value, which made it convenient to obtain comparison results. The GF value of the resistance under pressure is 0.5 , which is much lower than the GF under stretching (2.02-7.62). The GF value of the capacitance under pressure is 3.01, which is much higher than the GF under stretching (0.49). It can be revealed that the significant distinction of sensitivity ensures that the proposed sensor possesses excellent decoupling ability. In addition, the layer-bylayer casting process is suitable for large-scale manufacturing. The sensitivity of different batches of samples can be seen in Fig. S2 and Table S1, and the sensitivity of different samples is stable.

To investigate the real-time reliability of the SRCSM sensor, the relative resistance and capacitance variation ratios $\left(\Delta R / R_{0}, \Delta C / C_{0}\right)$ at stretching strains of $30 \%, 60 \%$, $90 \%$, and $120 \%$ and pressures of $2 \mathrm{kPa}, 4 \mathrm{kPa}$, and $14 \mathrm{kPa}$ under four loading-unloading cycles are depicted in Fig. 5. Each loading and unloading stimulus lasted for $20 \mathrm{~s}$ to investigate the stability of the sensor. It can be 

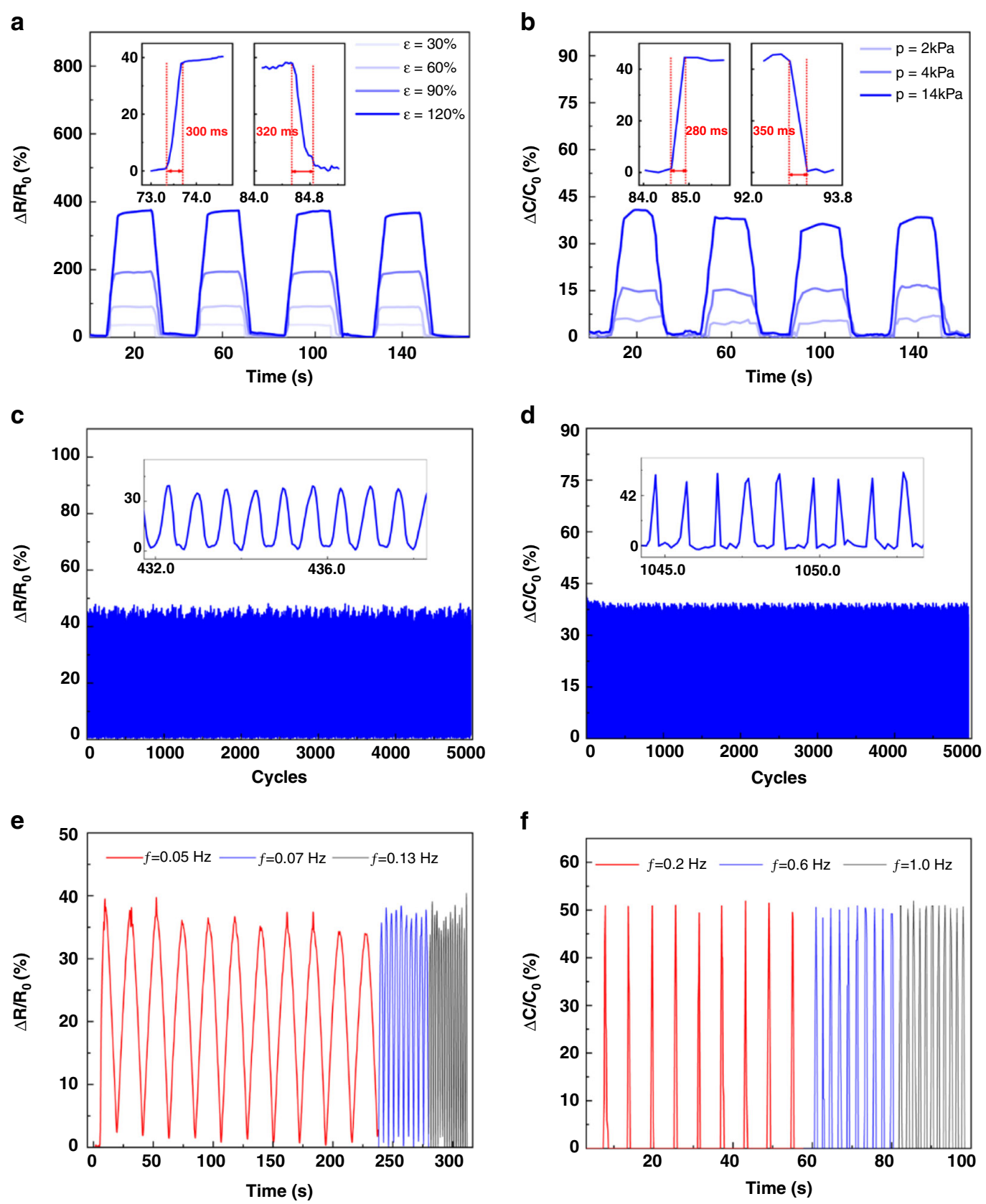

Fig. 5 Stability and reliable performance of SRCSM sensors. a Real-time relative resistance variation ratios $\left(\triangle R / R_{0}\right)$ of the SRCSM sensor under stretching strain. $\mathbf{b}$ Real-time relative capacitance variation ratios $\left(\triangle C / C_{0}\right)$ of the SRCSM sensor under pressure. The insets: The response and recovery times. c Durability test of the SRCSM sensor under 30\% strain during 5000 cycles with a stretching loading rate of $200.1 \mathrm{~mm} / \mathrm{min}$. $\mathbf{d}$ Durability test of the SRCSM sensor under $14 \mathrm{kPa}$ pressure for 5000 cycles with a pressure loading rate of $0.5 \mathrm{~mm} / \mathrm{min}$. e Dynamic responses of the SRCSM sensor with frequency changes from 0.05 to $0.13 \mathrm{~Hz}$ under $30 \%$ strain, $\mathbf{f}$ Dynamic responses of the SRCSM sensor with the changed frequency from 0.2 to $1.0 \mathrm{~Hz}$ under $14 \mathrm{kPa}$

observed that $\Delta R / R_{0}$ and $\Delta C / C_{0}$ gradually increased with increasing loaded stimuli (the stretching strains rose from $30 \%$ to $120 \%$, and the pressures increased from 2 $\mathrm{kPa}$ to $14 \mathrm{kPa})$. Moreover, $\Delta R / R_{0}$ and $\Delta C / C_{0}$ were maintained at stable values under each applied stimulus. These results indicate that the SRCSM sensor can monitor $\Delta R / R_{0}$ and $\Delta C / C_{0}$ under stimuli simultaneously. The insets in Fig. 5a, b illustrate that the response and recovery times are approximately $300 \mathrm{~ms}$ under a stretching strain of $30 \%$ and a pressure of $14 \mathrm{kPa}$, indicating a fast stimulus response, which can be satisfied to monitor human motions ${ }^{26-30}$. 
Stability and durability are essential for applications of wearable sensors. Figure $5 \mathrm{c}, \mathrm{d}$ shows the $\Delta R / R_{0}$ of the SRCSM sensor under $30 \%$ strain and $\Delta C / C_{0}$ of the SRCSM sensor under $14 \mathrm{kPa}$ pressure during 5000 cycles. $\Delta R / R_{0}$ and $\Delta C / C_{0}$ were stable with good repeatability during the whole durability test. The inset shows the stable signal output during the testing process. Furthermore, due to the highly tough overall structure, the utilized sensor also offers good dynamic performance in reproducibility and stability under different loaded stimuli frequencies, as shown in Fig. 5e, f. More details about the $\Delta R / R_{0}$ performance under pressure and $\Delta C / C_{0}$ performance under strain are shown in Fig. S3.

Table 1 compares the essential aspects of the multimode sensors reported in recent literature with our work. From the comparison, it can be declared that most single devices cannot collect and decouple multiple parameters simultaneously. Additionally, the assembly of two devices increases the complexity of the fabrication process. In contrast, our SRCSM sensor exhibits excellent decoupling capability under the premise of convenient manufacture and low cost. More comparison information is listed in Table S2, which indicates that the presented SRCSM sensor has excellent overall performance.

Application in Human Motion Monitoring and Recognition

To demonstrate the great potential applications of the proposed SRCSM sensor in the wearable electronic field, the real-time physical signals of the joint bending (finger, elbow, wrist, and knee) caused by human motions are measured. As shown in Fig. 6, the states of different joint activities are monitored by two sensor modes, and each action is distinguished by both $\Delta C / C_{0}$ and $\Delta R / R_{0}$ simultaneously. Figure 4 shows that the $\Delta R / R_{0}$ of the SRCSM sensor mainly depends on the stretching strain, while $\Delta C /$ $C_{0}$ depends on the pressure. For the elbow joint, due to the protrusion of the joint bone and the large skin surface stretch, $\Delta \mathrm{R} / \mathrm{R} 0$ and $\Delta C / C_{0}$ are very large, which belongs to the High $\Delta R / R_{0}$-High $\Delta C / C_{0}$ state. For the finger joint, the protrusion of the bone causes the sensor to be compressed when the finger joint is bent, resulting in a relatively large $\Delta C / C_{0}$. The small area of the stretching strain makes $\Delta R / R_{0}$ smaller than that of the elbow joint and wrist joint. Thus, finger joint bending corresponds to a low $\Delta R / R_{0^{-}}$high $\Delta C / C_{0}$ state. Since there is no protruding bone, the $\Delta C / C_{0}$ of the wrist joint is mainly affected by stretching strain, which is relatively small. The stretching strain of skin also leads to a significant $\Delta R / R_{0}$. It can be classified as a High $\Delta R / R_{0}$-Low $\Delta C / C_{0}$ state. For the knee joint, due to the apparent curvature of the kneecap even when laid flat, the conformally adhered sensor on the kneecap cannot detect enough strain, and $\Delta R / R_{0}$ and

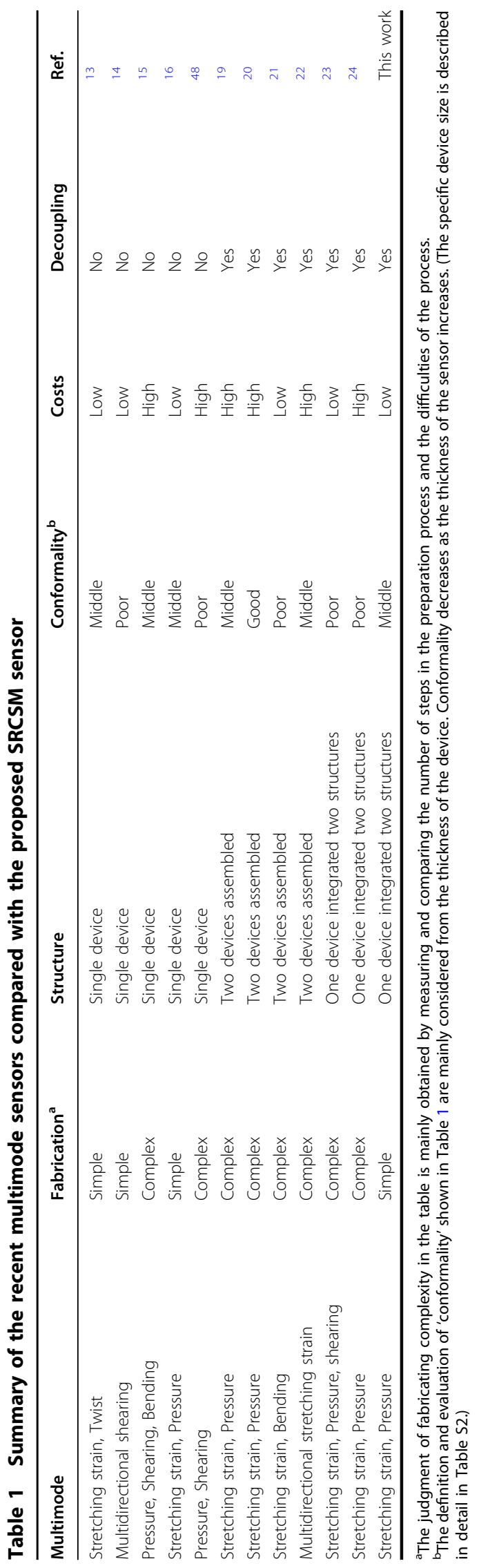



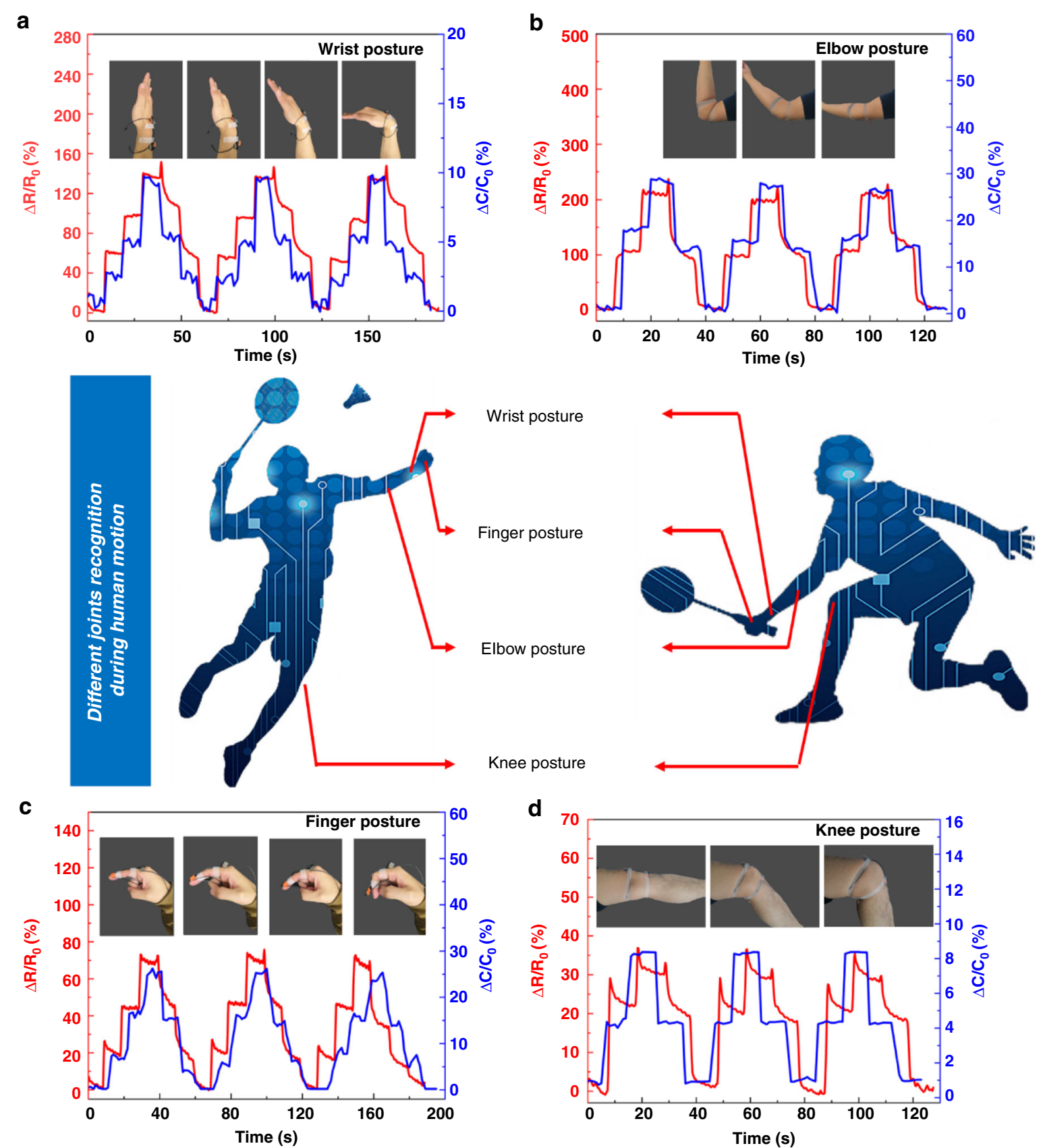

Fig. 6 Demonstrations of potential applications of the SRCSM sensor over a wide pressure and strain range. a Response of the sensor under $30^{\circ}, 60^{\circ}$, and $90^{\circ}$ bending of the wrist. $\mathbf{b}$ Response of the sensor under $45^{\circ}$ and $90^{\circ}$ bending of the elbow. $\mathbf{c}$ Response of the sensor under $30^{\circ}, 60^{\circ}$, and $90^{\circ}$ bending of the finger. $\mathbf{d}$ Response of the sensor under $45^{\circ}$ and $90^{\circ}$ bending of the knee

$\Delta C / C_{0}$ are both small during the motion of the knee joint, which is consistent with Low $\Delta R / R_{0}$-Low $\Delta C / C_{0}$ state corresponds.

Furthermore, due to the decoupled dual signal output characteristics of the SRCSM sensor, we applied the long short-term memory (LSTM) deep-learning algorithm to classify the electronic signals of different postures with different joints for recognition training. The LSTM neural network introduces the mechanism of the gating units based on the recurrent neural network (RNN) ${ }^{31-33}$. The gating mechanism controls the forgetting, input, and output of information, solving long-term dependence ${ }^{34-36}$. Figure $7 \mathrm{a}$ shows a schematic illustration of the artificial neural network algorithms. The input size of the LSTM layer is 2, corresponding to the input resistance and capacitance data, the number of hidden layers is 1 , and the 


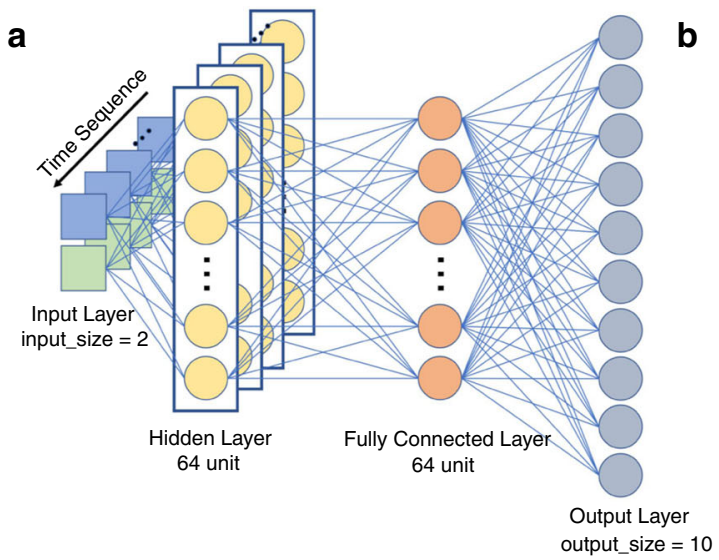

b

Joint posture confusion matrix (Resistance)

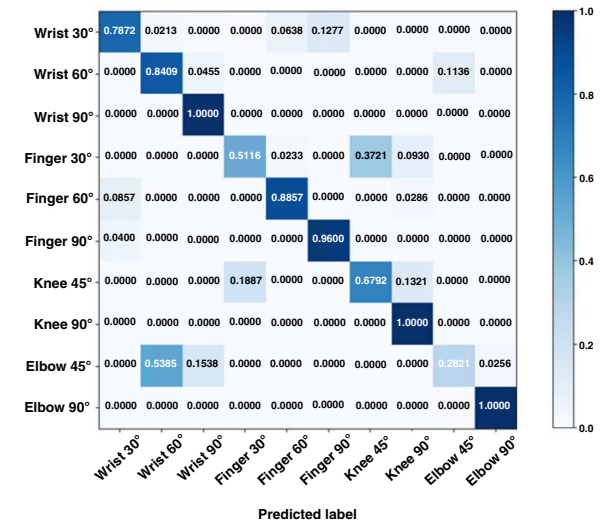

C

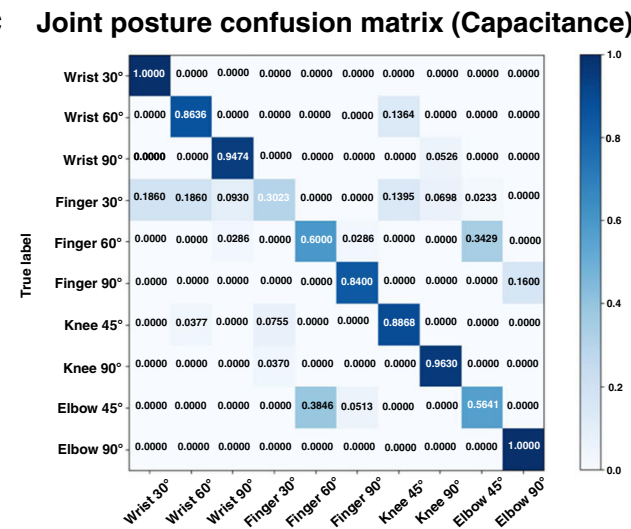

d Joint posture confusion matrix (Resistance \& Capacitance)

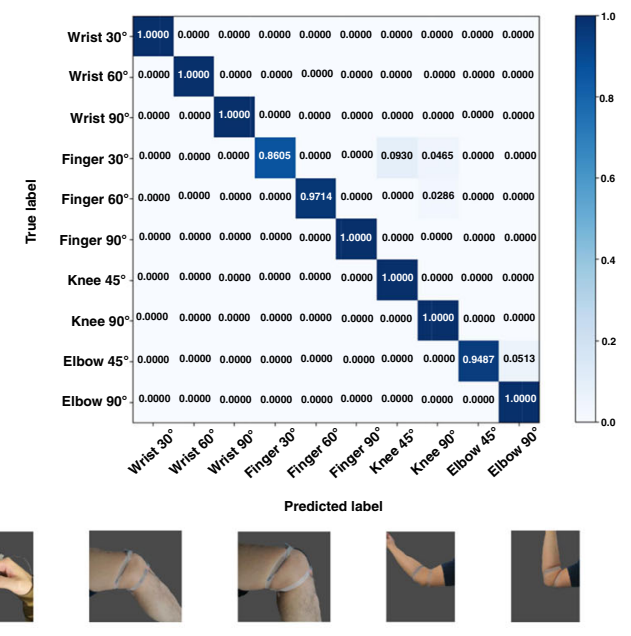

Classification accuracy using different data

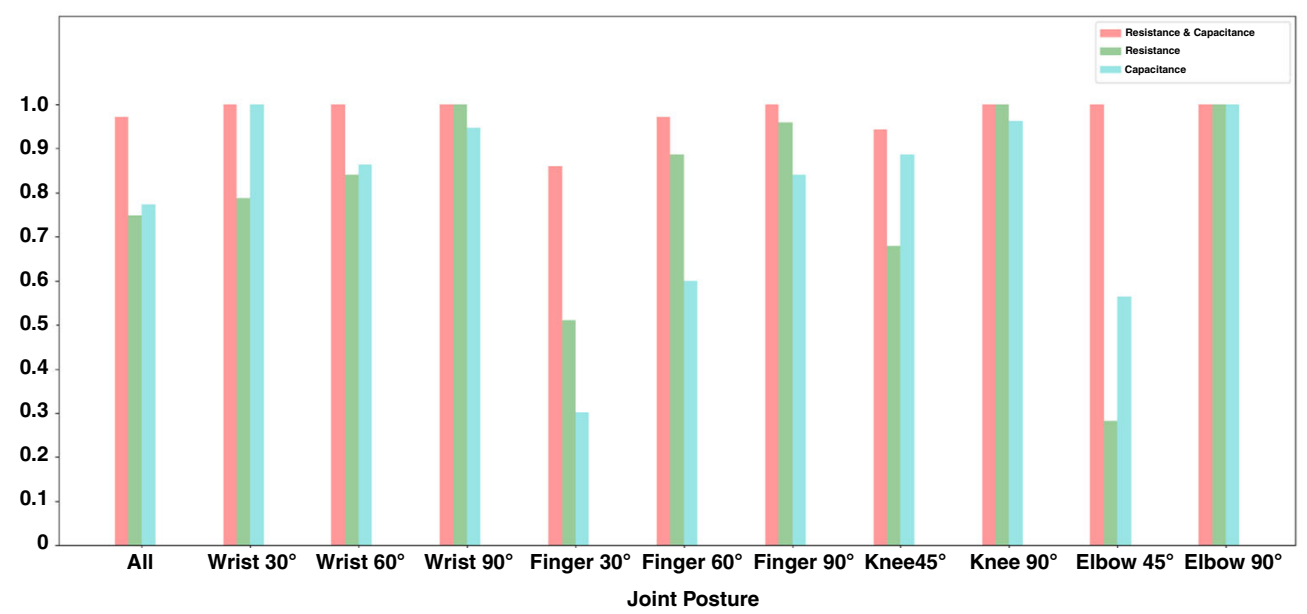

Fig. 7 Application in different postures recognition. a Schematic illustration of the long short-term memory (LSTM) deep-learning algorithm. b-d Classification confusion matrices using only resistance data, capacitance data, and both resistance and capacitance data. e Histogram of classification accuracy of all joint postures using different data 
output size is 10 , corresponding to 10 categories (wrist $30^{\circ}$, $60^{\circ}, 90^{\circ}$; finger $30^{\circ}, 50^{\circ}, 90^{\circ}$; knee $45^{\circ}, 90^{\circ}$, and elbow $45^{\circ}$, $90^{\circ}$ ). The softmax layer receives the output of the fully connected layer and completes the classification of the samples. The training set and the test set were divided at a ratio of 4:1, and the classification performance was evaluated based on the classification accuracy. Figure $7 \mathrm{~b}-\mathrm{d}$ shows the classification confusion matrix results using only resistance data, only capacitance data, and both resistance and capacitance data, respectively. In the case of using only electrical resistance data, the LSTM classifier does not perform well for the classification of wrist joints $30^{\circ}$, wrist joints $60^{\circ}$, finger joints $30^{\circ}$, knee joints $45^{\circ}$, and elbow joints $45^{\circ}$, and the classification accuracy is less than $85 \%$. When only the capacitance data are used, the classifier has a poor classification effect for finger joints $30^{\circ}$, finger joints $60^{\circ}$, and elbow joints $45^{\circ}$. A large number of elbow joint samples of $45^{\circ}$ are misclassified as knee $60^{\circ}$. It is speculated that the compressing degree of the elbow joint $45^{\circ}$ sensor is similar to that of the finger joints $60^{\circ}$, and the classification accuracy is less than $56.4 \%$. The results show that it is challenging to distinguish postures using a single capacitance or resistance signal effectively. When using both resistance and capacitance data, the classification accuracy of finger joint $30^{\circ}$, knee joint $45^{\circ}$, and elbow joint $45^{\circ}$ is $94.64 \%, 97.78 \%$, and $94.44 \%$, respectively. Moreover, the classification accuracies of other joint postures reach $100 \%$, which is significantly improved. Figure 7e displays the classification accuracy for all joint poses, and the highest classification accuracy is achieved by using both datasets. The overall classification accuracy rates of using only resistance data, capacitance data, and both resistance and capacitance data are $74.79 \%$, $77.36 \%$, and $97.13 \%$, respectively, which indicates that the SRCSM sensor has the highest classification accuracy in distinguishing postures.

Through the above analysis, the joint types and the bending degree of joints during motions can be distinguished by two independent output signals simultaneously from the SRCSM sensor. It has a meaningful advantage in scientific sports training, innovative medicine, and other fields. For example, while playing badminton, the signal of different joints can be used to recognize different postures, such as smashing, cutting, driving, and so on. Different sports, such as playing badminton and cycling, make the corresponding changes in different joints entirely different.

Due to the outstanding sensing performance, the proposed sensor not only detects large deformations but also monitors subtle motions such as voice recognition and pulse signals. The value of the relative resistance changes for different words and sentences is comparable with the existing research ${ }^{37-39}$. Additionally, the voice recognition system exhibits almost identical voltage waveforms and frequency responses during the repeatability tests, demonstrating high reproducibility and reliability. For pulse motion, characteristic peaks of the three human sphygmic waveforms ${ }^{40-43}$ relevant to percussion waves (P-waves), tidal waves ( $T$-waves), and diastolic waves ( $\mathrm{D}$-waves) can be distinguished. The peak of relative resistance changes is nearly $0.5 \%$, which shows superior performance compared with other studies ${ }^{44-47}$. Detailed information is shown in Fig. S4, demonstrating that the SRCSM sensor has outstanding performance in real-time monitoring of subtle motion areas.

\section{Conclusion}

In summary, we demonstrated a wearable integrated SRCSM sensor based on a low-cost and convenient layerby-layer casting fabrication process. This multimode sensor was integrated into a unique seamless structure, which simultaneously consisted of two main parts to decouple the different stimuli by an independent resistance-capacitance sensing mechanism. Benefitting from this excellent decoupling capability, the sensor can differentiate the motion characteristics of the positions and states of different joints with precise recognition (97.13\%) with the assistance of deep learning algorithms. In addition, subtle signals such as vocalization and pulse beats can also be detected due to the high sensitivity of the resistive component. Although this study is only a proof of concept demonstration, we anticipate that this simple, lost-cost but efficient strategy could be a prospective candidate for wearable applications in portable electronic skin, health care and intelligent sports monitoring devices, advanced human-machine interfaces, and intelligent soft robot perception systems.

\section{Experimental section \\ Materials}

MWCNTs with an average diameter of $50 \mathrm{~nm}$ and a length less than $10 \mu \mathrm{m}$ were purchased from XFNANO Inc. (Nanjing, China). Ecoflex was obtained from Smooth-On, Inc. (Macungie, U.S). The conductive metal tape was brought from MaoYe Inc. (Shenzhen, China). All the materials were utilized as received without any further purification.

\section{Characterization}

The morphology of the SRCSM sensor was observed via SEM (Zeiss Ultra Plus) at an acceleration voltage of $5 \mathrm{kV}$. The mechanical measurements were accomplished by a Mark-10 machinal testing machine (ESM303 force test stands and SERICE 5 digital force gauges). Electrical measurements were tested by a setup test system, including an LCR digital bridge (TH2826, TONGHUI) and a semiconductor parameter analyzer (Keithley 4200-SCS). 


\section{Acknowledgements}

We acknowledge the funding provided by the National Key Research and Development Program of China (Grant No. 2020YFB2008502), Jiangsu Provincial Natural Science Foundation of China (Grant No. BK20201268), and National Natural Science Foundation of China (Grant No. 12174050).

\section{Competing interests}

The authors declare no competing interests.

Supplementary information The online version contains supplementary material available at https://doi.org/10.1038/s41378-022-00358-2.

\section{Received: 11 September 2021 Revised: 2 December 2021 Accepted: 13} January 2022

Published online: 17 February 2022

\section{References}

1. Liu, Y. X., Li, H. Z. \& Zhang, M. Wireless battery-free broad-band sensor for wearable multiple. Physiological Meas. ACS Appl. Electron Meas. 3, 1681-1690 (2021).

2. Yang, H. T. et al. Wireless Ti3C2TX MXene strain sensor with ultrahigh sensitivity and designated working windows for soft exoskeletons. ACS Nano 14 11860-11875 (2020)

3. Zhang, S. D. et al. Ultrasensitive and highly compressible piezoresistive sensor based on polyurethane sponge coated with a cracked cellulose nanofibril/ silver nanowire layer. ACS Appl. Mater. Inter. 11, 10922-10932 (2019).

4. Wang, H. C. et al. High-performance foam-shaped strain sensor based on carbon nanotubes and Ti3C2TX MXene for the monitoring of human activities. ACS Nano 15, 9690-9700 (2021).

5. Zeng, X. W. \& Hu, Y. F. Sensation and perception of a bioinspired flexible smart sensor system. ACS Nano 15, 9238-9243 (2021).

6. Araromi, O. A. et al. Ultra-sensitive and resilient compliant strain gauges for soft machines. Nature 587, 219-224 (2020).

7. Yao, S. S. \& Zhu, Y. Wearable multifunctional sensors using printed stretchable conductors made of silver nanowires. Nanoscale 6, 2345-2352 (2014).

8. Ho, D. H. et al. Crack-enhanced microfluidic stretchable E-skin sensor. ACS Appl. Mater. Inter. 9, 44678-44686 (2017).

9. Li, Y., Luo, S. D., Yang, M. C., Liang, R. \& Zeng, C. C. Poisson ratio and piezoresistive sensing: a new route to high-performance $3 \mathrm{D}$ flexible and stretchable sensors of multimodal sensing capability. Adv. Funct. Mater. 26, 2900-2908 (2016)

10. Oh, J. et al. Pressure insensitive strain sensor with facile solution-based process for tactile sensing applications. ACS Nano 12, 7546-7553 (2018).

11. Yoo, J. Y. et al. Industrial grade, bending-insensitive, transparent nanoforce touch sensor via enhanced percolation effect in a hierarchical nanocomposite film. Adv. Funct. Mater. 28 https://doi.org/10.1002/ adfm.201804721 (2018)

12. Ge, J. et al. A stretchable electronic fabric artificial skin with pressure-, lateral strain-, and flexion-sensitive properties. Adv. Mater. 28, 722-728 (2016).

13. Wang, Z. Y. et al. Star-nose-inspired multi-mode sensor for anisotropic motion monitoring. Nano Energy $\mathbf{8 0}$ https:/doi.org/10.1016/j.nanoen.2020.105559 (2021).

14. Wang, C. et al. A directional strain sensor based on anisotropic microhoneycomb cellulose nanofiber-carbon nanotube hybrid aerogels prepared by unidirectional freeze drying. Small $\mathbf{1 5}$ https://doi.org/10.1002/ smll.201805363 (2019).

15. Won, S. M. et al. Multimodal sensing with a three-dimensional piezoresistive structure. Acs Nano 13, 10972-10979 (2019).

16. Song, Y. Q. et al. Carbon nanotube-modified fabric for wearable smart electronic-skin with exclusive normal-tangential force sensing ability. Adv. Mater. Technol.-US 4 https://doi.org/10.1002/admt.201800680 (2019).

17. Qin, Z. H. et al. Carbon nanotubes/hydrophobically associated hydrogels as ultrastretchable, highly sensitive, stable strain, and pressure sensors. ACS Appl. Mater. Inter. 12, 4944-4953 (2020).

18. Zhang, C. et al. Dopamine-triggered hydrogels with high transparency, selfadhesion, and thermoresponse as skinlike sensors. ACS Nano 15 1785-1794 (2021).
19. Boutry, C. M. et al. A stretchable and biodegradable strain and pressure sensor for orthopaedic application. Nat. Electron 1, 314-321 (2018).

20. Tas, M. O. et al. Highly stretchable, directionally oriented carbon nanotube/ PDMS conductive films with enhanced sensitivity as wearable strain sensors. ACS Appl. Mater. Inter. 11, 39560-39573 (2019).

21. Su, X. R. et al. Integrated wearable sensors with bending/stretching selectivity and extremely enhanced sensitivity. Chem. Eng. J. $\mathbf{3 8 9}$ https://doi.org/10.1016/ j.cej.2020.124503 (2020).

22. Lee, J. H. et al. Highly aligned, anisotropic carbon nanofiber films for multidirectional strain sensors with exceptional selectivity (vol 29, 1901623, 2019). Adv. Funct. Mater. 29 https://doi.org/10.1002/adfm.201901623 (2019).

23. Peng, S. H. et al. Multimodal capacitive and piezoresistive sensor for simultaneous measurement of multiple forces. ACS Appl. Mater. Inter. 12 22179-22190 (2020)

24. Park, S. et al. Stretchable energy-harvesting tactile electronic skin capable of differentiating multiple mechanical stimuli modes. Adv. Mater. 26, 7324-7332 (2014).

25. Nie, M. et al. Highly sensitive and large range strain sensor based on synergetic effects with double conductive layer structures. Sens. Actuat A-Phys. 318, 112515 (2021).

26. Pillay, J. D., Kolbe-Alexander, T. L., Proper, K. I., van Mechelen, W. \& Lambert, E. V. Steps that count: physical activity recommendations, brisk walking, and steps per minute-how do they relate? J. Phys. Act. Health 11, 502-508 (2014).

27. Eslami, M., Damavandi, M. \& Ferber, R. Association of navicular drop and selected lower-limb biomechanical measures during the stance phase of running. J. Appl. Biomech. 30, 250-254 (2014).

28. Boyer, K. A., Kiratli, B. J., Andriacchi, T. P. \& Beaupre, G. S. Maintaining femoral bone density in adults: how many steps per day are enough? Osteoporos. Int. 22, 2981-2988 (2011)

29. Tudor-Locke, C. et al. How Many Steps/day are Enough? For Adults. Int. J. Behav. Nutr. Phy. 8, doi:Artn 79 https://doi.org/10.1186/1479-5868-8-79 (2011).

30. Tudor-Locke, C. et al. How many steps/day are enough? for older adults and special populations. Int. J. Behav. Nutr. Phy. 8, doi:Artn 80 https://doi.org/ 10.1186/1479-5868-8-80 (2011).

31. Sun, B., Liu, M. Q., Zheng, R. H. \& Zhang, S. L. Attention-based LSTM network for wearable human activity recognition. Chin. Contr. Conf., 8677-8682 (2019).

32. Han, Y. K. \& Choi, Y. B. Human action recognition based on LSTM model using smartphone sensor. Int. Conf. Ubiq. Futur., 748-750 https://doi.org/10.1109/ icufn.2019.8806065 (2019).

33. Ke, W. S., Huang, D. X., Yang, F. \& Jiang, Y. H. Soft Sensor Development and Applications Based on LSTM in Deep Neural Networks. 2017 IEEE Symposium Series on Computational Intelligence (Ssci), 3468-3473 (2017).

34. Ordonez, F. J. \& Roggen, D. Deep convolutional and LSTM recurrent neural networks for multimodal wearable activity recognition. Sensors-Basel 16, do: ARTN 115 https://doi.org/10.3390/s16010115 (2016).

35. Zhou, X. K. et al. Deep-learning-enhanced human activity recognition for internet of healthcare things. IEEE Internet Things 7, 6429-6438 (2020).

36. Erdas, C. B. \& Guney, S. Human activity recognition by using different deep learning approaches for wearable sensors. Neural Process Lett. 53, 1795-1809 (2021).

37. Song, X. et al. A graphene-coated silk-spandex fabric strain sensor for human movement monitoring and recognition. Nanotechnology 32, doi: ARTN 215501 https://doi.org/10.1088/1361-6528/abe788 (2021).

38. Li, Y. X., Jiang, C. J. \& Han, W. H. Extending the pressure sensing range of porous polypyrrole with multiscale microstructures. Nanoscale 12 2081-2088 (2020)

39. Liu, P. J. \& Chen, W. H. Microwave-assisted selective heating to rapidly construct a nano-cracked hollow sponge for stretch sensing. J. Mater. Chem. C. 8, 9391-9400 (2020).

40. Ma, J. H. et al. Highly sensitive and large-range strain sensor with a selfcompensated two-order structure for human motion detection. Acs Appl Mater. Inter 11, 8527-8536 (2019).

41. Chen, W. Liu, L. X., Zhang, H. B. \& Yu, Z. Z. Kirigami-inspired highly stretchable, conductive, and hierarchical Ti3C2Tx MXene films for efficient electromagnetic interference shielding and pressure sensing. Acs Nano 15, 7668-7681 (2021).

42. Lee, J. et al. Ultrasensitive strain sensor based on separation of overlapped carbon nanotubes. Small 15, doi:ARTN 1805120 https://doi.org/10.1002/ smll.201805120 (2019).

43. Qiu, J. et al. Rapid-response, low detection limit, and high-sensitivity capacitive flexible tactile sensor based on three-dimensional porous dielectric layer for wearable electronic skin. Acs Appl Mater. Inter 11, 40716-40725 (2019). 
44. Si, Y. et al. Ultralight biomass-derived carbonaceous nanofibrous aerogels with superelasticity and high pressure-sensitivity. Adv. Mater. 28, 9512-+ (2016).

45. Yin, B. et al. Highly stretchable, ultrasensitive, and wearable strain sensors based on facilely prepared reduced graphene oxide woven fabrics in an ethanol flame. Acs App/ Mater. Inter 9, 32054-32064 (2017).
46. linear response over exceptionally broad pressure range. Acs Nano 12, 4045-4054 (2018).

47. Wang, C. Y. et al. Carbonized silk fabric for ultrastretchable, highly sensitive, and wearable strain sensors. Adv. Mater. 28, 6640-+ (2016).

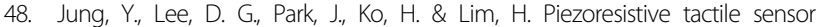
discriminating multidirectional forces. Sens.-Basel 15, 25463-25473 (2015). 